\title{
Article \\ Comparative Transcriptomic Analysis of the Hematopoietic System between Human and Mouse by Single Cell RNA Sequencing
}

\author{
Shouguo Gao *, Zhijie Wu, Jeerthi Kannan, Liza Mathews (D), Xingmin Feng (D), Sachiko Kajigaya and Neal S. Young \\ Hematology Branch, NHLBI, National Institutes of Health, Bethesda, MD 20892, USA; zhijie.wu@nih.gov (Z.W.); \\ jeerthi.kannan@nih.gov (J.K.); liza.mathews@nih.gov (L.M.); xingmin.feng@nih.gov (X.F.); \\ sachiko.kajigaya@nih.gov (S.K.); youngns@nhlbi.nih.gov (N.S.Y.) \\ * Correspondence: shouguo.gao@nih.gov
}

\section{check for} updates

Citation: Gao, S.; Wu, Z.; Kannan, J.; Mathews, L.; Feng, X.; Kajigaya, S.; Young, N.S. Comparative Transcriptomic Analysis of the Hematopoietic System between Human and Mouse by Single Cell RNA Sequencing. Cells 2021, 10, 973. https://doi.org/10.3390/ cells10050973

Academic Editors: Tuhin Subhra Santra and Fan-Gang Tseng

Received: 10 March 2021

Accepted: 19 April 2021

Published: 21 April 2021

Publisher's Note: MDPI stays neutral with regard to jurisdictional claims in published maps and institutional affiliations.

Copyright: (c) 2021 by the authors. Licensee MDPI, Basel, Switzerland. This article is an open access article distributed under the terms and conditions of the Creative Commons Attribution (CC BY) license (https:// creativecommons.org/licenses/by/ $4.0 /)$.
Abstract: (1) Background: mouse models are fundamental to the study of hematopoiesis, but comparisons between mouse and human in single cells have been limited in depth. (2) Methods: we constructed a single-cell resolution transcriptomic atlas of hematopoietic stem and progenitor cells (HSPCs) of human and mouse, from a total of 32,805 single cells. We used Monocle to examine the trajectories of hematopoietic differentiation, and SCENIC to analyze gene networks underlying hematopoiesis. (3) Results: After alignment with Seurat 2, the cells of mouse and human could be separated by same cell type categories. Cells were grouped into 17 subpopulations; cluster-specific genes were species-conserved and shared functional themes. The clustering dendrogram indicated that cell types were highly conserved between human and mouse. A visualization of the Monocle results provided an intuitive representation of HSPC differentiation to three dominant branches (Erythroid/megakaryocytic, Myeloid, and Lymphoid), derived directly from the hematopoietic stem cell and the long-term hematopoietic stem cells in both human and mouse. Gene regulation was similarly conserved, reflected by comparable transcriptional factors and regulatory sequence motifs in subpopulations of cells. (4) Conclusions: our analysis has confirmed evolutionary conservation in the hematopoietic systems of mouse and human, extending to cell types, gene expression and regulatory elements.

Keywords: hematopoiesis; gene regulatory network; single-cell RNA sequencing; cross-species analysis

\section{Introduction}

Hematopoiesis is a stepwise process originating from hematopoietic stem cells (HSCs) associated, functionally, with loss of self-renewal, activation of lineage specific transcription factors (TFs), and upregulation of downstream genes for progenitor cells and their mature progenies [1-3]. From multipotent progenitors, the common lineages for myelopoiesis (common myeloid progenitor, CMP) and lymphopoiesis (common lymphoid progenitor, CLP) are segregated. During myeloid differentiation, oligopotent CMPs undergo further restriction into bivalent granulocyte-monocyte progenitors (GMPs) of granulocytes and monocytes, and megakaryocyte-erythroid progenitors (MEPs) that terminate in platelets and red blood cells [4]. The classical model of hematopoiesis was a tree, rooted in the long-term hematopoietic stem cell (LTHSC) and branching into multipotent, oligopotent, and unipotent progenitors. However, novel single cell approaches, which can profile gene expression of thousands of individual cells, have challenged established models of hematopoiesis [5,6].

Single cell RNA-sequencing (scRNA-seq) has been used to examine the conservation of transcription across species in various organs [7-11]. For example, scRNA-seq revealed the surprisingly well-conserved cellular architecture between human and mouse brains: similarity in hierarchical organization, corresponding relationships at the subclass level 
and no major missing homologous classes [10]. In pancreas, scRNA-seq analysis showed major cell types (alpha, beta, gamma, delta, ductal and endothelial) to be conserved between mouse and human [7]. Shay $\mathrm{T}$ et al. observed that the expression patterns of most orthologous genes are conserved in the immune system, but several hundreds of genes showed clearly divergent expression between human and mouse [12]. In our previous study, co-expression and regulatory networks of hematopoietic genes were well conserved between human and mouse. The co-expression network showed "small-world" and "scalefree" architectures. The gene regulatory network formed a hierarchical structure and hematopoiesis transcription factors localized to the hierarchy's middle level, and, also, tended to participate in blood-related diseases [13]. While scRNA-seq has been extensively applied to investigate hematopoiesis in human and mouse, cross-species comparisons of hematopoietic hierarchy have not been intensively reported, or appear only as one component of larger reports, and are relatively superficial. For example, in an early study, lineage transcriptomic characteristics were shown to be similar, without description of differentiation trajectories and consideration of transcription networks [11].

In the present work, we utilized the $10 \times$ single cell platform and a canonical correlation analysis (CCA) computational strategy, and we conducted a comparative transcriptomic analysis of the hematopoietic hierarchy in human and mouse. We found that the hematopoietic stem and progenitor cell (HSPC) compartments in the two species are composed of populations characterized by the same sets of homologous genes, and the hematopoietic lineages and transcriptional profiling in hematopoiesis are well conserved between human and mouse. Lineage specificity and maturation were mainly determined by transcription factors and their target genes. We developed a comprehensive atlas for cell type-specific regulatory sequence motifs and TF-centered regulatory networks (regulons). The primary sequence motifs of most cell type-specific TFs and their target genes were conserved between human and mouse. Our results indicate evolutionary similarity in the human and mouse hematopoietic systems.

\section{Materials and Methods}

Bone marrow samples were obtained from healthy donors after written informed consent, in accordance with the Declaration of Helsinki, and following protocols approved by the National Heart, Lung, and Blood Institute (NCT00001620). CD3 ${ }^{-}$CD14 ${ }^{-}$CD19 ${ }^{-}$CD34 ${ }^{+}$ cells were sorted using a LSRII Fortessa Cytometer (BD Biosciences, San Jose, CA, USA). Lineage $^{-} \mathrm{CD}_{117^{+}}$cells were sorted from bone marrow of C57BL/6 mice (Figure 1a). The Chromium Single Cell $3^{\prime}$ platform $(10 \times$ Genomics) was used to prepare scRNA-seq cDNA libraries [14,15]. RNA-seq libraries were sequenced with paired-end reads of 75-bp on Illumina HiSeq 3000 System. The cellranger pipeline (https:/ / support.10xgenomics.com/ single-cellgene-expression/software/pipelines/latest/what-is-cell-ranger, accessed on 18 October 2018) was used to process raw data, align reads to the genome, and generate gene-cell expression matrices. Specifically, sequencing reads of human were aligned to the hg19 reference genome by STAR and uniquely aligned reads were calculated to quantitate gene expression levels for all ENSEMBL genes with unique molecular identifiers (UMIs). Low-quality cells were filtered and removed from further analyses if the number of detected genes was fewer than 500 (due to low quality, potential fragments) or more than 3000 (due to potential doublets). Cells with a high percentage of mitochondrion gene reads $(>10 \%)$ were also excluded. Raw and processed data from all experiments were deposited in the NCBI Gene Expression Omnibus with GSE135194 and GSE142235 [14,15]. Downstream analyses were performed using the R software package Seurat (http:/ / satijalab.org/seurat/, v2.3.4, accessed on 18 June 2018). Raw reads in each cell were first scaled by a library size to 10,000 and then log-transformed. Highly variable genes $(\sim 1300$, identified with y.cutoff $=0.5)$ were used for Principal Component Analysis (PCA) of high-dimensional data. Top 30 principal components were selected for unsupervised clustering of cells with a graph-based clustering approach. Graph-based clusters methods were applied to group cells based on two-dimensional $t$-distributed Stochastic Neighbor Embedding (tSNE) using Seurat at 
resolution 2 [16]. Each gene from a cluster was compared to the median expression of the same gene from cells in all other clusters by FindMarkers function in Seurat and genes with $p<0.01$ were defined as cluster-specific genes [17]. Genes then were ranked based on their expression fold change, and top cluster-specific genes were compared with published cell type-specific genes [1,2]. An HSPC subtype was assigned to each cluster based on statistical significance of overlap between HSPC- and cluster-specific genes (with smallest $p$ values of Fisher's exact test) [17]. Sequencing reads of mouse were aligned to the $\mathrm{mm} 9$ reference genome by STAR and the gene-cell expression matrix was calculated. Subsequently, gene expression analysis for mouse followed the same pipeline as for human, using cell lineage specific genes derived from GSE81682 in GEO as references for cell type assignment.

We used the canonical correlation analysis (CCA) algorithm to perform comparative transcriptomic analysis of the hematopoietic system between human and mouse (RunCCA function in Seurat 2 with parameter of num.cc $=20$ ). The CCA algorithm is a multivariate statistical technique for the determination of linear associations between two sets of variables that are maximally correlated. In scRNA-seq analysis, the CCA algorithm can detect the statistical common factors between two digital gene expression (DGE) matrices, which vary due to batch effects or different methods used in normalization procedures. These factors are aggregations of conserved gene-to-gene correlations between human and mouse, and, therefore, we could align all human cells against all mouse cells in an identical linear space and visualize shared populations in different species with further analyses such as t-SNE.

To explore the conservation of cell populations between human and mouse, we applied scmap (http:/ / bioconductor.org/packages/scmap, accessed on 10 July 2019) to project cells from a human scRNA-seq dataset onto cell types defined in the mouse scRNA-seq dataset, with a parameter threshold of 0.7 , and, conversely, from human to mouse datasets. Cells in human were expected to be projected to the closest mouse cell types if there was transcriptional conservation.

Differentiation trajectory analyses were conducted with Monocle (https://www. bioconductor.org/packages/release/bioc/html/monocle.html, accessed on 6 June 2019). Preprocessed Seurat objects were imported into Monocle with the "importCDS" function. Monocle's "orderCells" function arranged cells along a pseudo-time axis to indicate their position in a developmental continuum. The trajectory trees identified by Monocle were colored by cell types or expression levels of marker genes to show the differentiation directions during hematopoiesis. The reversed graph embedding algorithm in Monocle 2 was used to impute differentiation trajectories in both 2- and 3-dimensions.

With RcisTarget software in Bioconductor, we identified putative transcription factor motifs; SCENIC algorithm was used to construct gene networks and model regulon activity within each cell state. We followed the developer's instructions (https://github.com/ aertslab/SCENIC, accessed on 18 May 2019) pipeline for these analyses. For each cell type-specific gene list of human and mouse, we scanned two motif TFs databases (using RcisTarget in Bioconductor on hg19-tss-centered-10 kb-7species.mc8nr.feather or mm9tss-centered-10 kb-7species.mc9nr.feather from https:/ / resources.aertslab.org/cistarget/, accessed on 10 April 2019) and retained modules with significant motif enrichment [18]. 
a

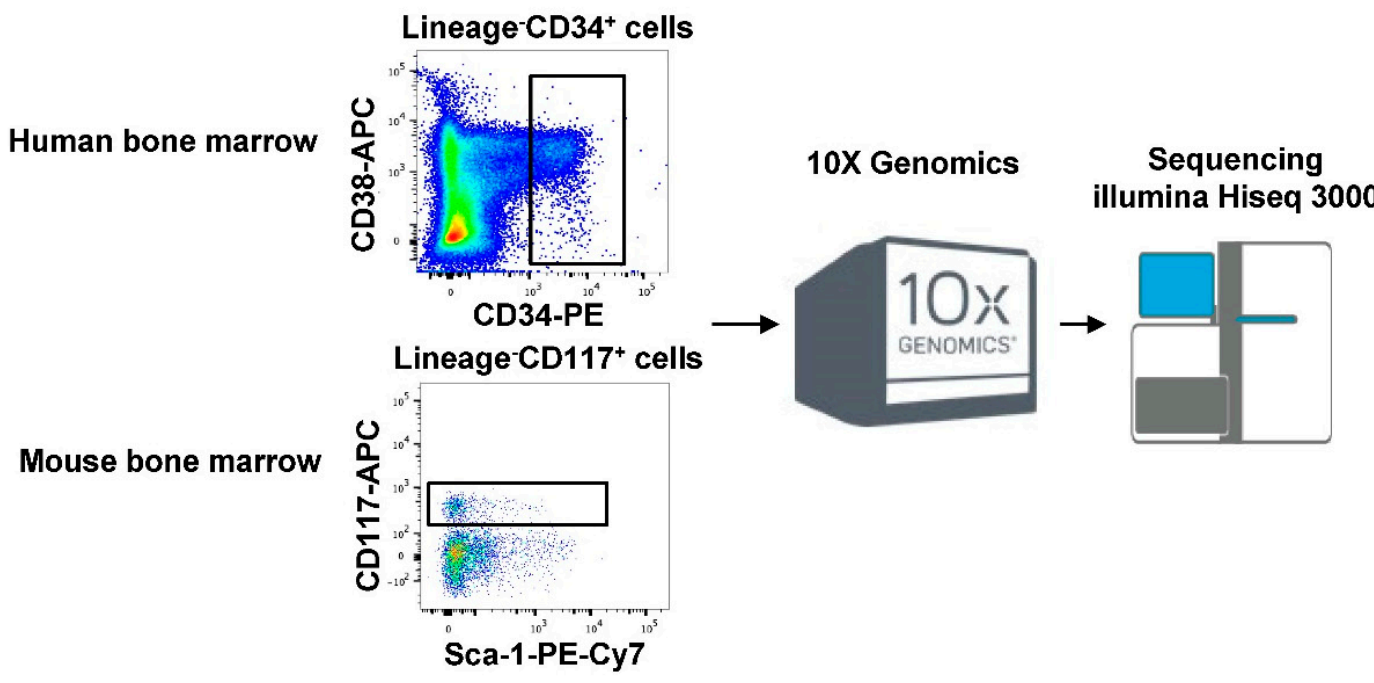

b

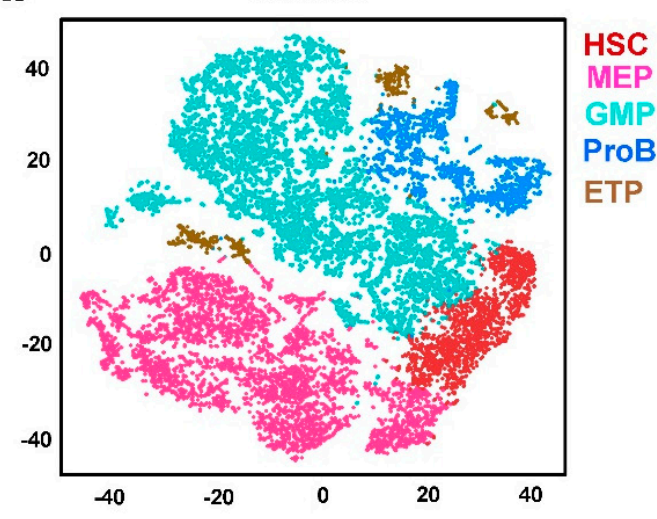

C

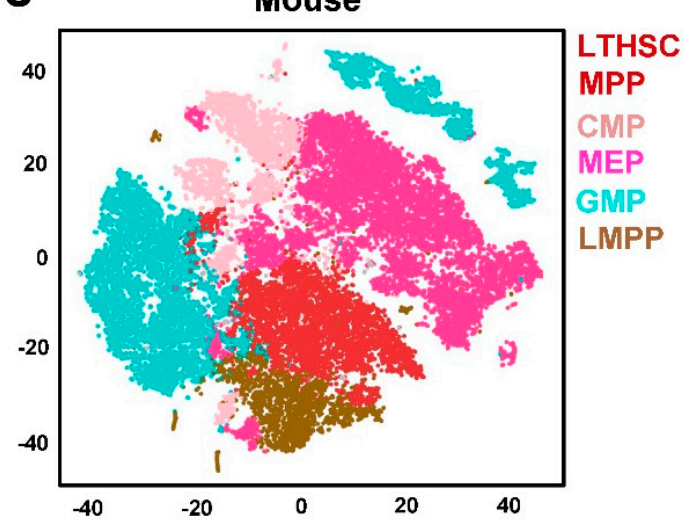

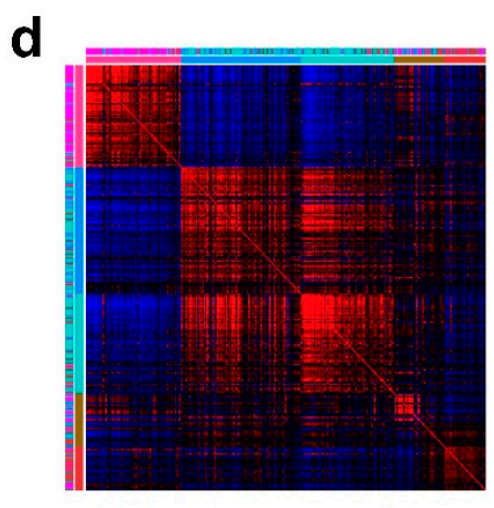
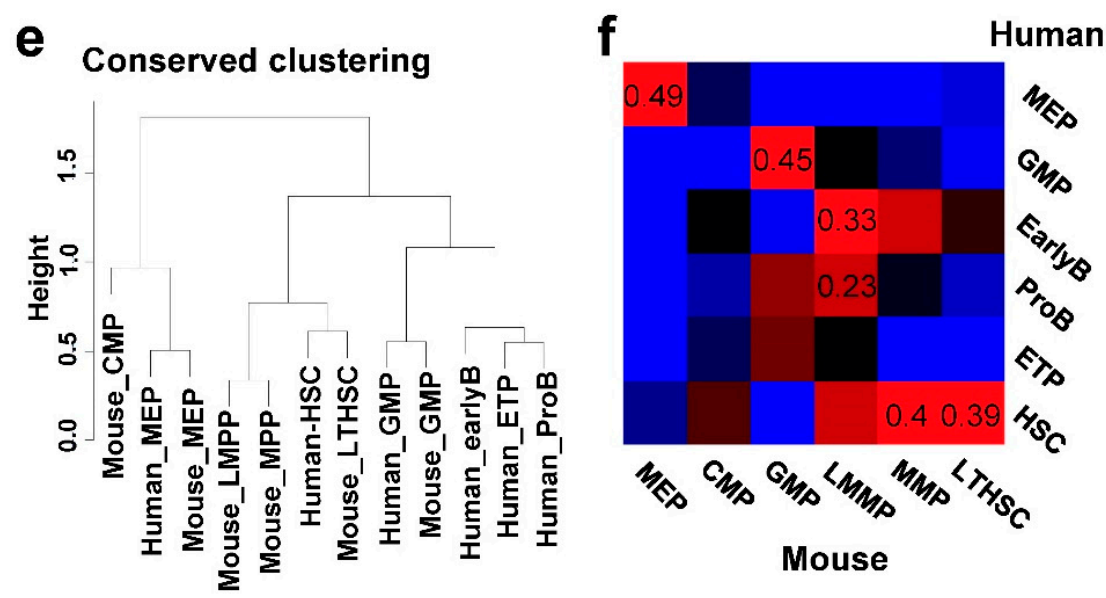

Figure 1. (a) Schematic overview of the study design. (b) A tSNE plot of single-cell gene expression of human HSPCs. (c) A tSNE plot of single-cell gene expression of mouse HSPCs. (d) Correlation of expression between human and mouse orthologous cell-type specific genes (red indicates high correlation and blue indicates low correlation). Cell types of species (inner was for human) were marked by the colors defined in (b). Conservation of homologous genes between species was evident. (e) The phylogenetic tree of human and mouse cell populations. Average expression across human-mouse homologous genes was calculated for cell types of human and mouse, and the distance between expression patterns for different cell types was used for hierarchical clustering. (f) Correlation of expression levels of homologous genes in human and mouse cell populations. 
For regulon analysis, the expression matrices of human and mouse were first extracted from Seurat objects and then transformed into the required format for SCENIC, in which rows represented genes and columns represented cells [18]. Cells with less than 500 and more than 3000 detected genes were filtered. We also filtered genes with less than at least 6 UMI counts in human and mouse. We used GENIE3 in the Bioconductor expression matrix to identify co-expressed gene modules and infer potential TF targets for each module. Regulatory modules (regulons) were identified from co-expression and DNA motif analyses. Regulons were then evaluated in each cell to ascertain their activities by the AUCell package in Bioconductor, before a binary matrix (with 1 for active and 0 for inactive, with threshold determined by the distribution of AUCell scores) was obtained. To profile gene regulatory module features of all HSPCs, the Spearman correlation coefficients between regulons were calculated, and only the positive-correlated targets in the regulons were retained. We chose top5perTarget as a parameter of coexMethod to run SCENIC [18], such that the top 5 percent of TFs were employed to create regulons for each gene. Cell specificities of the identified regulons were assigned with Fisher's exact test using the mark gene list [1,2]. topGO was used to functionally annotate the identified regulons [19]. Networks of the TF regulons with motif information were visualized by Cytoscape [20].

\section{Results}

\section{1. scRNA-Seq Identified a Comprehensive and Conserved List of HSPC Types}

We obtained bone marrow samples from four healthy human donors. In order to characterize the early stages of hematopoiesis, we sorted lineage ${ }^{-}$CD $34^{+}$cells to enrich for HSPCs. After filtering out cells with limited numbers of detected genes, our dataset contained 15,245 single CD34 ${ }^{+}$stem/progenitor cells. Sequencing data of single CD34 ${ }^{+}$cells, as visualized tSNE (Figure 1b), displayed clear clusters, suggesting distinct cell types at the molecular level. Hematopoietic cell identity was assigned to each cell cluster by comparing cluster-specific genes with a reported lineage signature gene list [1,2]. CD34 ${ }^{+}$cells were clustered into 15 clusters and then could be computationally assigned to the following cell populations: multipotent progenitor HSCs, megakaryocyte-erythroid progenitors (MEPs), granulocyte-monocyte progenitors (GMPs), B lymphocyte progenitors (ProBs), and early $\mathrm{T}$ lineage progenitors (ETPs) (Figure 1b). The number of clusters identified by Seurat depends on the resolution selected. Although resolutions 1, 2 and 3 generated different numbers of clusters, cell type assignment at each resolution was almost identical and did not affect further analyses.

For analysis of murine hematopoiesis, 17,560 linage ${ }^{-} \mathrm{CD} 117^{+}$cells from B6 mice were also clustered, unsupervised, based on transcriptome similarity using tSNE (Figure 1c). Hematopoietic cell identity was assigned to each cluster of cells by comparing clusterspecific genes with accepted lineage signatures. We could group the cells into 36 clusters and then assign them into long-term hematopoietic stem cells (LTHSC), multipotent progenitors (MPP), lymphoid multipotent progenitors (LMPP), common myeloid progenitors (CMP), MEP, and GMP.

We defined HSC in human and MPP and LTHSC in mouse as conserved HSC; ProB in human and LMPP in mouse as lymphoid cells; and GMP and MEP in both species as GMP and MEP, respectively. We considered that these were species comparable/closest cell populations [21,22].

We analyzed human and mouse datasets in parallel with orthologous genes in InParanoid [23]. tSNE plots showed cells grouped by species, instead of by a cell type, due to species specificity and batch effects (Figure S1a, human and mouse cells were profiled at different times). The CCA algorithm is a multivariate statistical technique for finding linear associations between two sets of variables that are maximally correlated. In scRNA-seq analysis, the CCA algorithm can detect the statistical common factors among two digital gene expression (DGE) matrices, which vary from each other due to batch effects or different methods used in normalization procedures. After alignment with CCA, cells of mouse and human were well mixed and separated into the same cell type categories 
(Figure S1b,c). The cells clustered into 17 subpopulations $(15,245,17,560$ cells, and 17, 16 subpopulations contain cells from human and mouse, respectively) by computational analysis (Figure S1c). Cell assignments were validated from expression of typical cell type-specific genes (Figure S1d). Complete gene lists in different cell populations were shown in Supplemental File 1. There were many homologous genes apparent in the two gene lists for the same cell types of human and mouse. The top 25 specific genes and their expression are shown in Figure 2, in which the homologous genes were highlighted and linked with lines. Typical hematopoiesis-related genes and their expression in different cell populations are shown in Figure S2.

\subsection{Conserved Cell-Type Expression between Human and Mouse}

We built a human-to-mouse one-to-one orthologous gene list (13,520 genes), collected from InParanoid (http:/ /inparanoid.sbc.su.se, accessed on 16 August 2018) for homology analysis [23]. Matching cell types requires shared expression patterns between species, and we found that there were many homologous genes that best discriminated mouse and human HSPCs. Identification of homologous types or classes enabled an analysis of conservation and divergence of gene-expression patterns between the two species. For each pair of homologous types, we compared the expression of orthologous genes and investigated cell type-specific genes between human and mouse. There were a high number of genes in human and mouse which shared the same cell-type specificity (Rand index $=0.24, p<0.00001$, Supplemental File 1), especially in MEP and HSC. There were many divergent genes that only express in certain lineages of human, not in those of mouse, and vice versa (Supplemental File 1). We collected the overlap of type-specific gene lists in human and mouse, calculated averages of gene-to-gene correlations with human and mouse datasets, and conducted a clustering analysis with the averaged correlations (Figure 1d). The column annotations for the assigned cell types of marker genes in human (inner) and mouse (outer) showed high consistency, implying a conservation of cell typespecific genes.

We explored similarities and differences in HSPCs between two species. To derive a quantitative view of the cellular evolution from mouse to human in hematopoiesis, we used mouse and human orthologous genes and calculated an average of expression of cells of each type for human and mouse. After hierarchical clustering (ward.D2 method, with 1-Pearson correlation as distance), a dendrogram indicated that cell types were highly conserved between the two species (Figure 1e). For example, the MEP of mouse and human shared very similar transcriptomes, and a GMP also showed high similarity. Human HSC were first clustered with mouse LTHSC by the nearest neighbor (Figure 1e), and then with mouse MPP. MPP and LMPP in mouse had a similar transcriptome, which was already observed in human [1]. The high correlations of human and mouse in the same cell types showed that cell-type similarity in orthologous gene expression dominated species differences, particularly for the MEP and HSC populations (Figure 1e), as observed by others $[8,18,24]$.

A heatmap in Figure 1f quantitatively presents the expression similarity of cell types in human and mouse. The mouse MEP cluster shows strong correlations with the human MEP $\left(\mathrm{R}=0.496, p<1 \times 10^{-10}\right)$, and the human HSC shows strong correlation with mouse LTHSC $\left(\mathrm{R}=0.389, p<1 \times 10^{-10}\right)$ and $\mathrm{MPP}\left(\mathrm{R}=0.395, p<1 \times 10^{-10}\right)$. Conservation of mammalian cell types in single-cell comparative genomic studies have been reported $[11,24]$. 
a
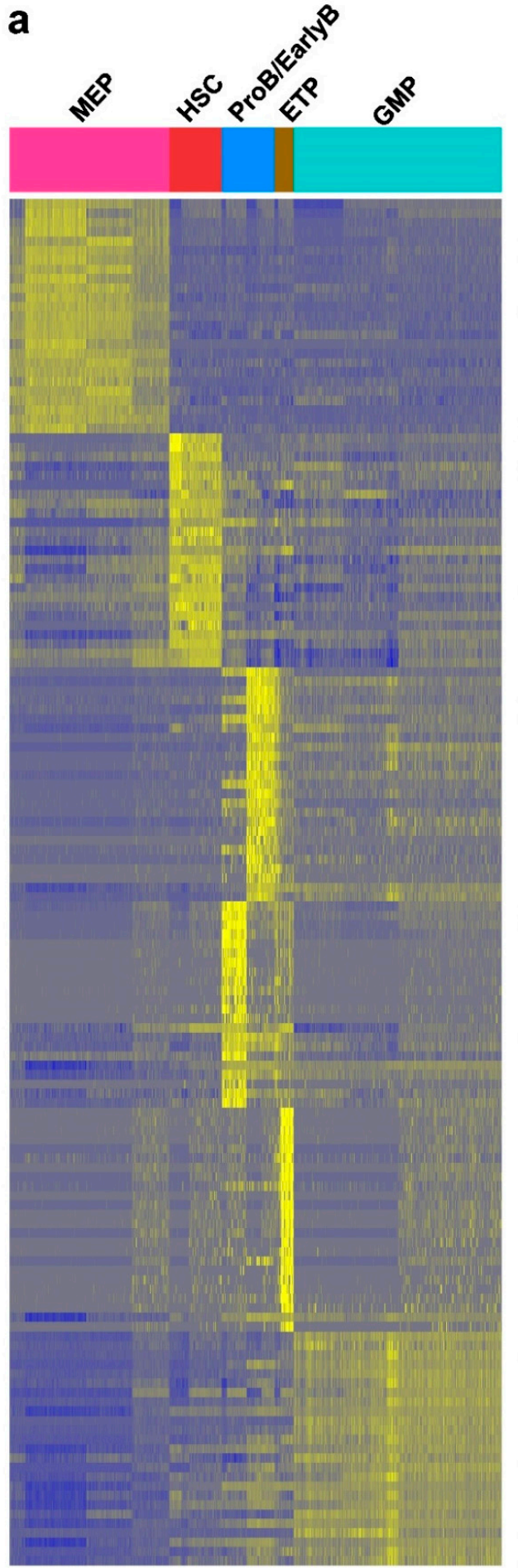

Human b

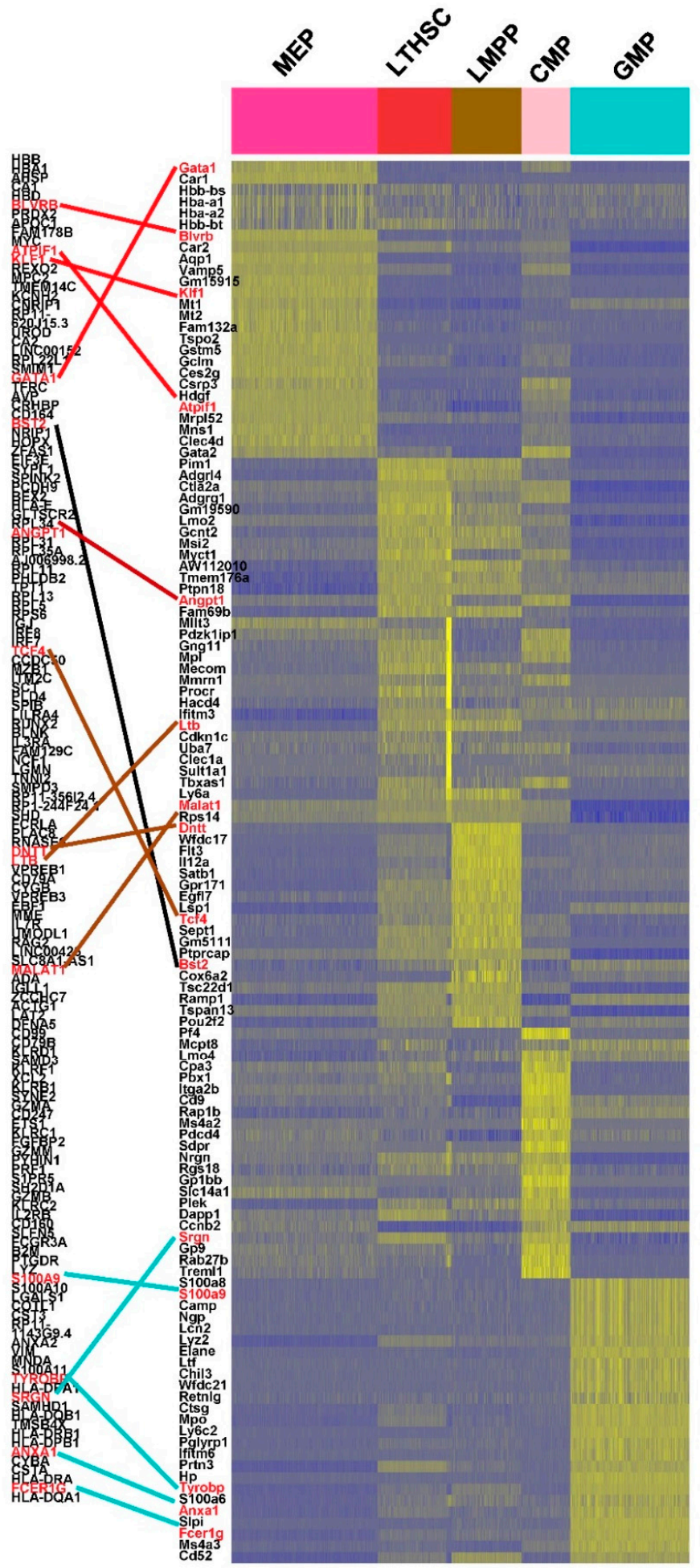

Mouse

Figure 2. Heatmaps of differentially expressed genes in (a) human and (b) mouse cell populations. Heatmaps showed a scaled gene expression of the top 25 genes representing each of the identified cell populations found in human and mouse. Each row represents one gene and each column displays gene expression of 5 pooled cells. Genes are listed in the middle and homologous genes were linked together (colored by the cell type specificity). The marker gene lists of concordant cell populations in human and mouse tend to be homologous. Note that MEP and GMP were well characterized, but others were not well characterized in human and mouse due to the characteristic of cell populations. 


\subsection{Projection by Scmap and Conservation of Cell Populations between Human and Mouse}

The sharing of marker genes shown in Supplemental File 1 and Figure 2 simply confirmed the comparable cell populations of human and mouse. To be more quantitative, single-cell transcriptomes of human cells were compared with those of mouse cells using scmap [25]. scmap projects a cell onto a reference dataset, allowing inference of cellular identity based on resemblance of transcriptomes to the reference cell type. Seurat was used to normalize gene expression by library sizes and $\log 2$ transformed the data, and libraries were subset by expression of shared genes across datasets. Most human MEP cells $(85 \%)$ were mapped to mouse MEP cell types based on transcriptional similarity, suggesting functional similarity and species conservation. A total of $48 \%$ of human HSC cells were mapped to mouse LTHSC cell types, and $24 \%$ were mapped to MPP, indicating the similarity of MPP and HSC (Figure 3a,b). Other human cell types were also mainly mapped to their closest murine cell types. The mapping results are consistent with known human-mouse comparable/closest cell populations [21,22]. In total, our analysis confirmed conservation of hematopoietic stem and progenitor cell types between human and mouse.

\subsection{Developmental Trajectories in Human and Mouse Hematopoiesis}

Clustering is based on an assumption of biologically distinct groups, such as discrete cell types or states; pseudo-temporal ordering assumes that data lie on a connected manifold [26]. For detailed analysis of the transition from stem cells to lineage-restricted progenitors, we used Monocle to arrange each cell by pseudo-temporal ordering based on gene expression [27]. After applying Monocle to the profiled human and mouse cells, an intuitive graphical representation of early stages of HSPC differentiation emerged. In human, lineages clearly separated among lineage ${ }^{-} \mathrm{CD} 34^{+} \mathrm{CD} 38^{+}$progenitors (Figure $3 \mathrm{c}$ ). We defined HSC in human and LTHSC in mouse as roots, so that they were located at starting points of the hierarchy. In both human and mouse, three branches arose from HSC and LTHSC. We confirmed cells in the three branches as erythroid/megakaryocytic, myeloid and lymphoid (Figure 3c,d). At the cellular level, the adjacency of cell types on plotting reflects differentiation pathways. The differentiation trajectories of human and mouse are highly similar, as described by others [1,2]. The newly defined model shows unexpected developmental shifts within the progenitor cell architecture: where many stem and progenitor cell types are multipotent, the stem cell compartment is multipotent and only progenitors are unipotent. These features do not present in the classic hierarchical model. We also examined expression level changes of individual genes within the trajectories. As an example, GATA1 expression increased along the erythroid branch, and GATA2 expression decreased with differentiation. There was a GATA1 and GATA2 switch in human and mouse, showing species conservation of gene participations during erythroid differentiation (Figure 3c,d) [28]. Expression changes of other representative lineage-specific genes along human and mouse differentiation trajectories also were shown in Figure 3c,d and Figure S3. Therefore, at both cellular and molecular/gene levels, human and mouse show conservation during differentiation. scRNA-seq allowed for deconvolution of heterogenous HSPC population, both LTHSC and CD34- as stem cells and lineagecommitted progenitors, and for the reconstruction of a trajectory of normal hematopoietic differentiation in human and mouse. Monocle 2 was also used to analyze the data and produce 2- and 3-dimensional projections, which were similar to those generated with the Monocle ICA algorithm (Figure S4) [29]. Conservation of hematopoietic differentiation between human and mouse was evident from the observations that genes were activated at the same differentiation stages across species [9], and both human and mouse cells were distributed along pseudo-temporally ordered paths from HSCs/LTHSCs to three branches_erythroid/megakaryocytic, myeloid, and lymphoid. 


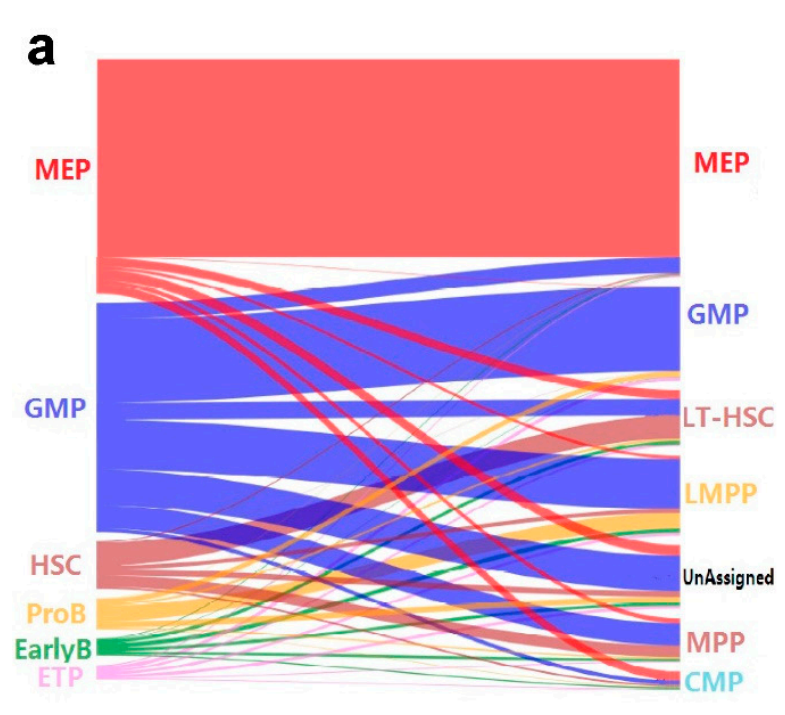

C Human
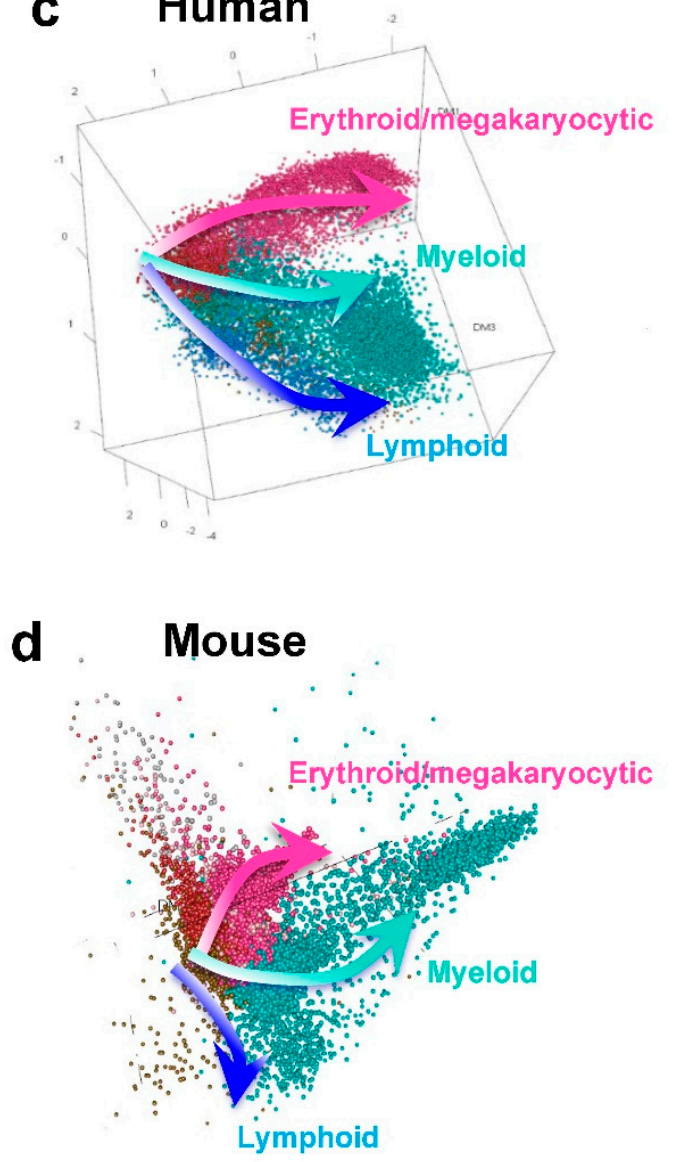

b

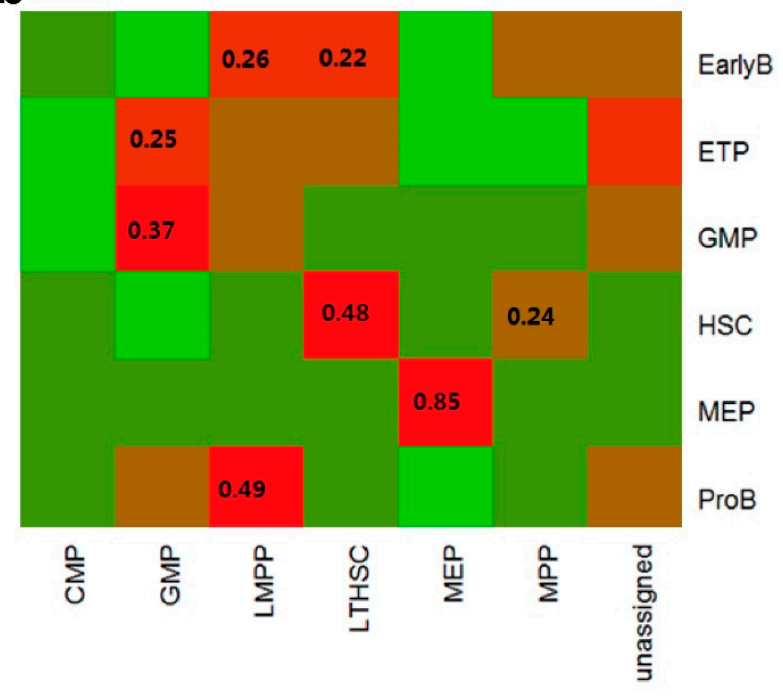

GATA1
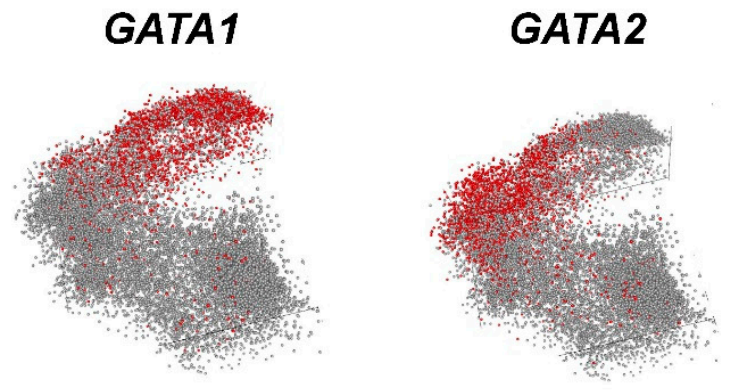

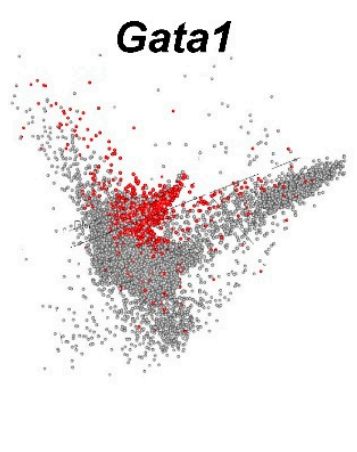

\section{Gata2}

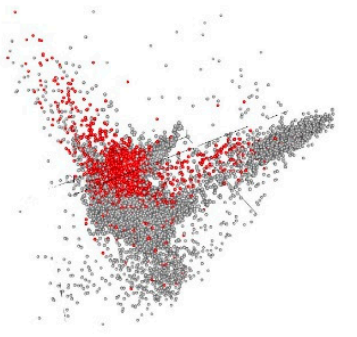

Figure 3. (a) Sankey diagrams of scmap-cluster projection of human dataset to mouse dataset. Each row is a cell of human, colored with cell types. (b) Fractions of human cell populations projected to mouse cell types. Cells tended to be projected to the corresponding cell population defined in mouse; for example, $85 \%$ of MEP mouse cells were mapped to human MEP cell types. (c,d) Monocle revealed linage differentiation trajectories of human (c) and mouse (d) HSPCs. Cells (balls, colored based on predicted cell type) are arranged in a 2D space calculated with Independent Component Analysis. The results obtained with Monocle 2 are shown in Figure S3, showing the same themes. Expression of GATA1 and GATA2 for lineages are highlighted in a differentiation tree, showing a clear GATA2 and GATA1 switch during differentiation. 


\subsection{Conserved Cell Type Specific Regulatory Elements/Motifs between Human and Mouse}

Given a gene list, the program RcisTarget identifies over-represented TF-binding motifs and can predict candidate target genes (regulons) based on databases containing motifs with genome-wide rankings. The over-representation of each motif for the gene list is estimated through calculating a normalized enrichment score (NES) by AUCell algorithm. After the cell type-specific gene lists were input for calculation, 1494 (in human) and 663 (in mouse) TFs with their recognition motifs were identified as significantly enriched in different cell populations. We found that there were species conservation of transcription factors and recognition motifs (Figure 4a) in the most similar cell types. As shown in Figure 4a, MEP gene-set was enriched for a GATA1 associated "cisbp_M0801" motif $(\mathrm{NES}=4.29)$, and this motif was also enriched in mouse with NES = 3.88. GMP gene-set was enriched for a CEBPA associated "cisbp_M0315" motif (NES = 6.95), and this motif was also enriched in mouse with NES $=3.76$.

The complete list of well-defined cell type-specific motifs, their corresponding transcription factors for human and mouse, as well as species conservation, are shown in Supplementary File 2. Some motifs showed species conservation and were active in only one cell population. Some motifs were species-specific, restricted in their appearance, only among human or mouse cell populations. Hematopoietic differentiation is controlled by key transcription factors (TFs), which regulate stem cell functions and differentiation. The same TFs in human and mouse tend to contribute to the same hematopoietic lineage differentiation, but some TFs only function in hematopoiesis of human or mouse. For example, in both human and mouse, higher activities of GATA1- and E2F1-related motifs were present in MEP cells; SRF and RELA motifs in GMP cells; IRF and CBFB motifs in lymphoid cells; and GATA2 and CHD1 motifs in HSC cells were observed. In addition, other TFs were identified in specific cell populations of both species, such as upregulation of FOSB, JUN, and JUND in HSC cells, and higher activity of JUN, JUNB, GTF2B and $C E B P D$ in lymphoid cells. Antagonism between transcription factor PU.1 (encoded by SPI1) and GATA2 drives myeloid/lymphoid versus erythroid/megakaryocyte lineage commitments $[30,31]$. In both human and mouse, GATA1 is the key transcriptional factor for erythro- and megakaryocytic differentiation; GATA2 downregulation and reciprocal GATA1 upregulation maintain cell differentiation in the erythroid/megakaryocytic lineage [30-32]. We observed higher activities of GATA1 motifs in MEP, while SPI1 motifs were exclusive in GMP cells of human and mouse. Transcription factor MEIS1 is an HSC marker, and its expression level declines with cell differentiation; it is known to promote expression of stem cell markers in leukemias [33,34]. We observed MEIS1 motifs exclusively activate in LTHSC and MPP of mouse, indicating higher activity in stem cells and early stage of multipotent progenitors. The cell type expression specificity of motifs is consistent with function. FOSB, JUN, JUND and JUNB are members of activator protein-1 (AP-1). AP-1 is involved in cell differentiation, proliferation and survival [35]. JUNB is required for Th17 cell development [36]. The expression of FOSB, together with GFI1, RUNX1 and SPI1, are sufficient to generate immunocompetent HSCs in adult mouse endothelial cells [37]. Progression from the CLP to B/Myeloid and ProB Precursors during B Lymphopoiesis requires CEBP. CEBPA is required for Flt $3^{+}$CLP maturation into ProB cells and then for proliferation [38]. Collectively, multiple TFs, alone and in combination, regulate hematopoietic stemness and differentiation. 
a

\begin{tabular}{|c|c|c|c|c|c|}
\hline gene set & motif & NES & AUC & specie & TF \\
\hline MEP & cisbp__M0801 & 3.88 & 0.079 & mouse & Gata1 \\
\hline MEP & cisbp_M0801 & 4.29 & 0.0531 & human & GATA1 \\
\hline GMP & cisbp_M0315 & 6.95 & 0.0671 & mouse & Cebpb \\
\hline GMP & cisbp_M0315 & 3.76 & 0.067 & human & CEBPA \\
\hline LTHSC & homer_AACAGGAAGT_Ets1-distal & 3.16 & 0.0776 & mouse & Elk1 \\
\hline HSC & $\begin{array}{c}\text { taipale_tf_pairs_ELK1_TEF_NSCGGA } \\
\text { WNTTACGTAAN_CAP }\end{array}$ & 3.31 & 0.0663 & human & ELK1 \\
\hline LMPP & taipale_RUNX3_full_NAACCRCAAN & 3.76 & 0.101 & mouse & Cbfb \\
\hline EarlyB & transfac_pro__M00769 & 3.59 & 0.0588 & human & CBFB \\
\hline
\end{tabular}

b

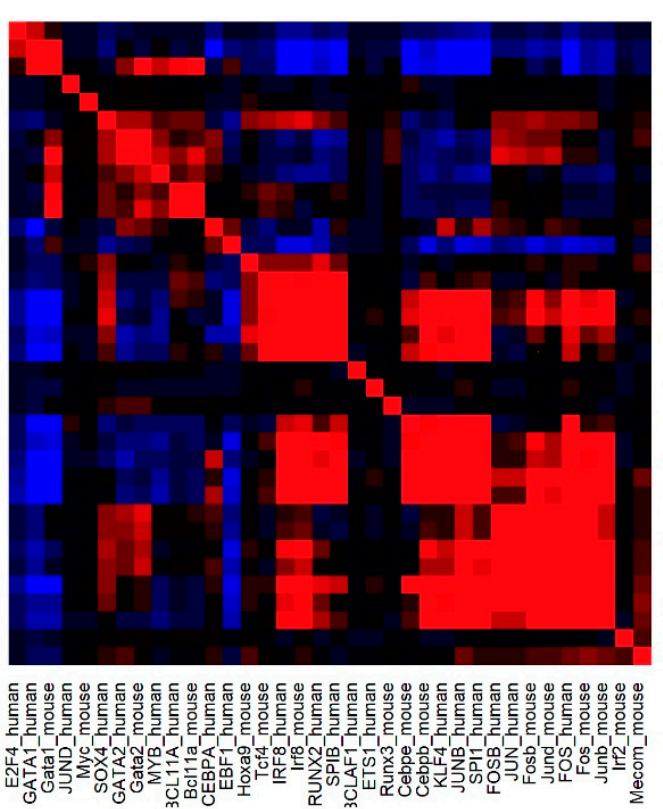

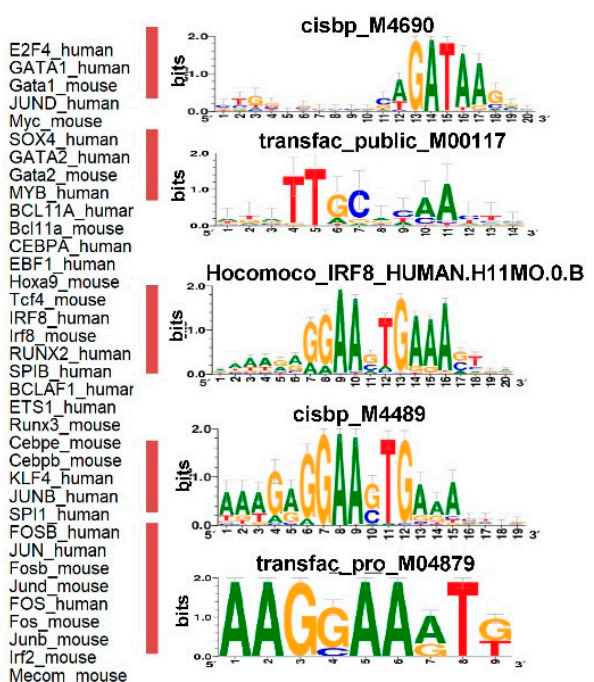

IIrt2-mouse

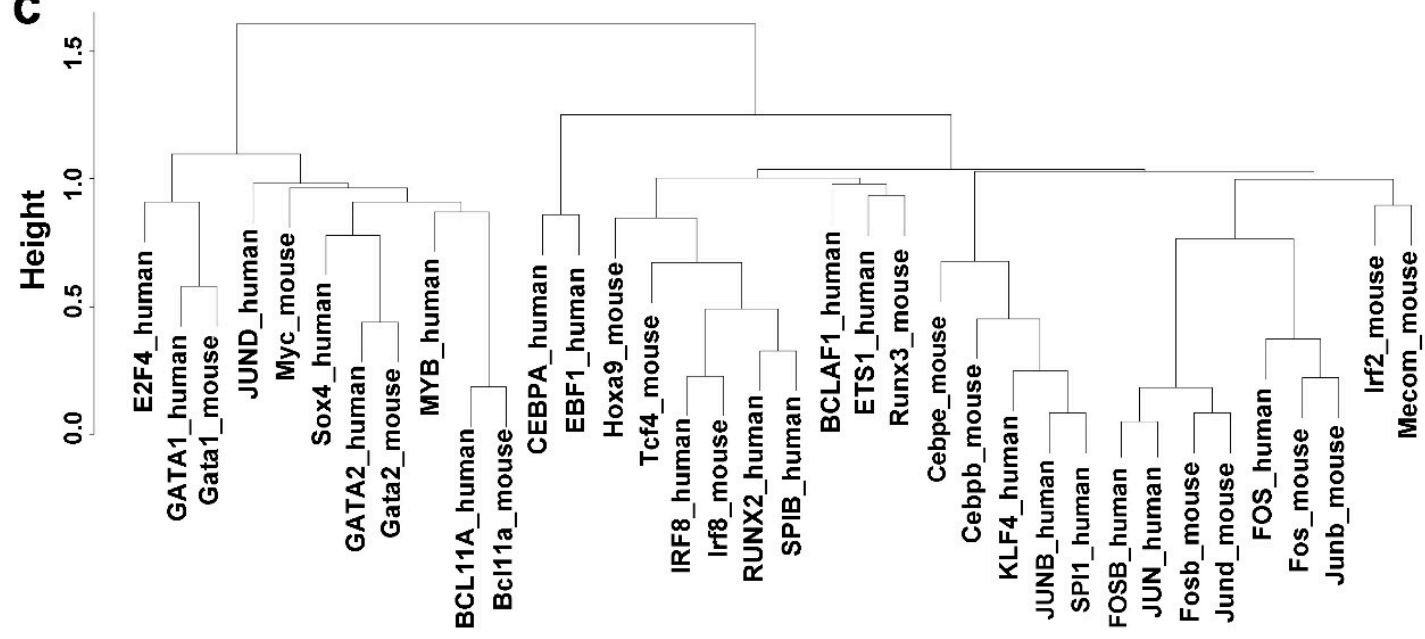

Figure 4. (a) A table of enriched motifs by RcisTarget for selected sets of regulons related to cell populations in human and mouse, generated within the SCENIC workflow. (b) Average AUCell scores of all human and mouse cells were calculated with the identified regulons to obtain a regulon-cell matrix. Then, the distance among regulons was hierarchically clustered. The corresponding binding motifs were shown on the right. Regulons were grouped into several major modules, along with representative TF regulons and associated cell types. (c) A clustering dendrogram shows the relationships of 37 orthologous TF regulons. 


\subsection{Conserved Cell Type Specific Regulatory Networks between Human and Mouse}

To recapitulate the gene regulatory relationships among the different populations, and thus infer the regulatory mechanisms underlying hematopoiesis, we used SCENIC, an algorithm to deduce Regulatory Networks and cellular status from scRNA data [18]. A cell expression matrix of 10,000 highly variable genes extracted from Seurat object (data slot after normalization) was imported as an input matrix for SCENIC. By identifying the coexpression modules (including transcriptional factors) and analysis of cis-regulatory motif analysis with each co-expression modules, we obtained 84 cell identity-specific regulons, together with their corresponding targets (Supplementary File 3). The number of targeted genes ranged from 4 to 587 (average, 63).

Next, we investigated the gene networks that underlie mammalian cell-type conservation. For example, the GATA1 regulons for MEP in human and GATA1 regulons for MEP in mouse were both identified as enriched regulons (Figure $4 b, c)$. We examined TF regulons from both human and mouse in SCENIC and identified 24 orthologous TF regulons, which could be grouped into five major modules across human and mouse (Figure $4 \mathrm{~b}$ and Supplementary File 3). As examples, GATA1 and E2F4 are associated with erythropoiesis, and IRF8 is associated with GMP populations. These modules were enriched not only for lineage-specific transcription factors but also for conserved binding motifs that lead to coordinated module activation in lineage commitment (Figure $4 \mathrm{~b}$, right). In contrast to a published work [24], we did not find much species-specific genetic regulation between human and mouse. We also clustered regulons based on a Pearson correlation of their AUCell scores in all cells (Figure 4c), and expanded to include extended motifs defined in SCENIC (Figure S5c). Again, clustering was driven by cell types rather than by species. This reflected that homologous genes played dominant roles in the clustering results. If non-homologous genes expressed in only human or mouse contribute heavily to variation, regulons would be clustered into two groups (human and mouse) rather than being grouped by the same/similar regulons across the two species.

We used the Fisher's exact test to check the cell type specificity of regulons, and the result is shown in Supplementary File 4. Briefly, to test the enrichment of a cell type annotation for a regulons, we used a One-tailed Fisher's exact test to determine the significance of the association between gene members in regulons and marker gene list, and thus determined the enrichment of cell type for the regulons [17]. Most regulons are cell type-specific, and the regulons associated with same transcriptional factors were assigned to the same cell populations in human and mouse. For example, GATA1 regulons were assigned to the MEP of human, and the MEP and CMP of mouse. IRF regulons were assigned to the GMP of human and mouse. Some regulons were not cell type-specific, such as SP1 and JUND. These regulons were active in many cell populations, indicating their importance in the whole stage of differentiation.

We also examined the potential regulatory role of novel transcription factors by a gene ontology (GO) enrichment analysis of target genes in 23 novel regulons. Functional annotations of regulons are provided in Supplementary File 5. Enriched GO terms for GATA1 are associated with the activation/differentiation of erythropoiesis terms GO:0030218 (erythrocyte differentiation) and GO:0034101 (erythrocyte homeostasis). YBX1 regulon was annotated with GO:0030218 (erythrocyte differentiation); $Y B X 1$ is a transcription factor widely expressed in all cellular lineages during differentiation and is involved in erythroid cell development [39]. Under regulation by GATA factors, YBX1 functions in erythroid differentiation and aberrant expression of $Y B X 1$ gene results in dyserythropoiesis [39,40]. IRF8 targeted genes were highly related to biological processes, such as immune response and lymphocyte activation, with terms of GO:0006955 (immune response), GO:0001775 (cell activation), and GO:0030217 (T cell differentiation). 
After more detailed exploration of the same-TF regulatory networks inferred for regulons between human and mouse, we found that there was a significantly higher number of conserved targets (evaluated by the Fisher's test for significance of overlap), and some species-specific targets genes, as reported by another study [18]. Two examples of such an analysis are shown in Figure S5a,b. For regulons generated with SCENIC using the target genes of IRF8 in human and mouse, the edges represent the connections between each of the two TFs and their target genes. Human and mouse shared 34 targeted genes, significantly higher than predicted by chance $\left(p<1 \times 10^{-60}\right.$, Fisher's test). For regulons generated with SCENIC using the targeted genes of GATA1 in human and mouse, there were seven shared targeted genes $\left(p<1 \times 10^{-10}\right.$, Fisher's test). Species conservation of hematopoiesis was apparent at the gene network level, both for TFs and their targets.

SCENIC uses the AUCell algorithm to score the activities of entire gene regulatory networks or regulons in each cell, which can be clustered and displayed in activity matrices (Figure 5a,b). To determine the "on/off" activity of each regulon, AUCell automatically identifies the threshold of activity and generates the binary activity regulon matrix ( 1 for active, 0 for inactive). Next, the t-SNE algorithm was used to project all cells onto the two dimensions, based on the binary regulon activity matrix, to examine whether it can accurately identify cellular types. Consistent with the Seurat tSNE algorithm, cell clustering using regulon activity clearly distinguished different cell populations (Figure $5 c, d$ ), confirming the characterization of cell identity by tSNE based on the activities of regulons by integrating the expression of TFs and their targets. Cell clustering using activities of regulons, rather than by gene expression, resulted in t-SNE projections to distinct cell populations. The activation of cell-type-specific networks was conserved between human and mouse (Figure $5 \mathrm{e}, \mathrm{f})$, which was evident from conserved regulon activities for human and mouse in the same cell populations. 

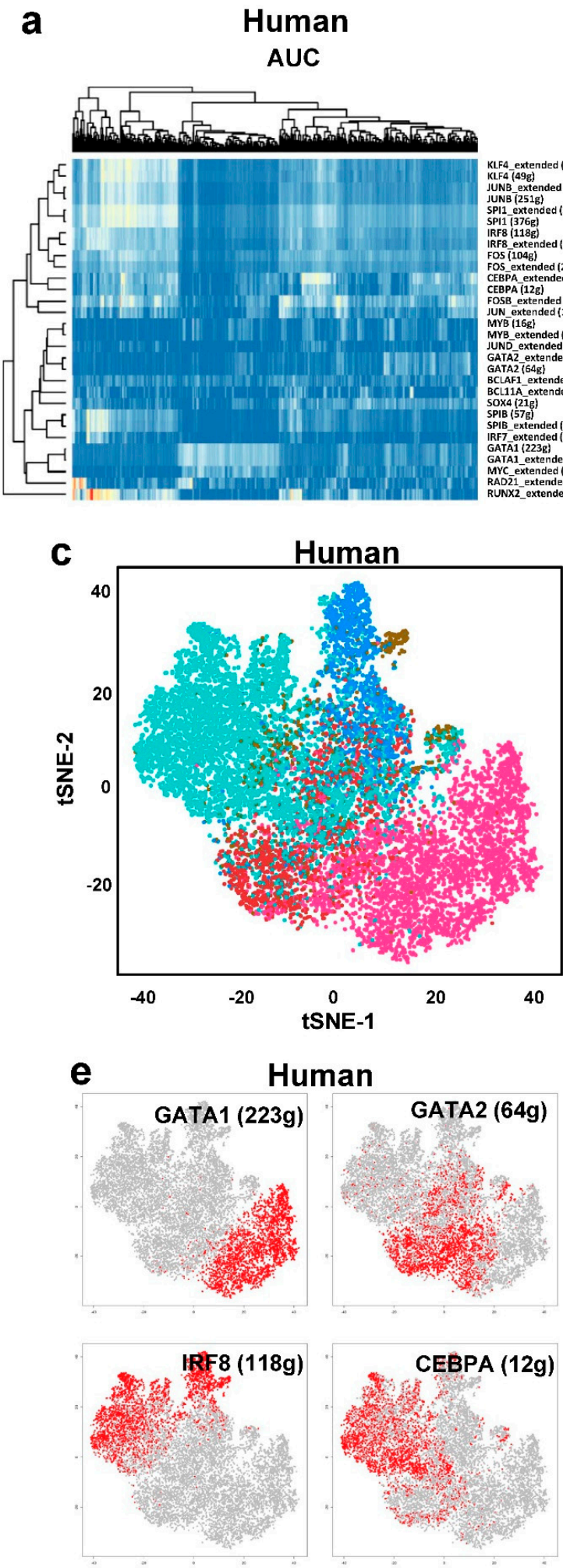
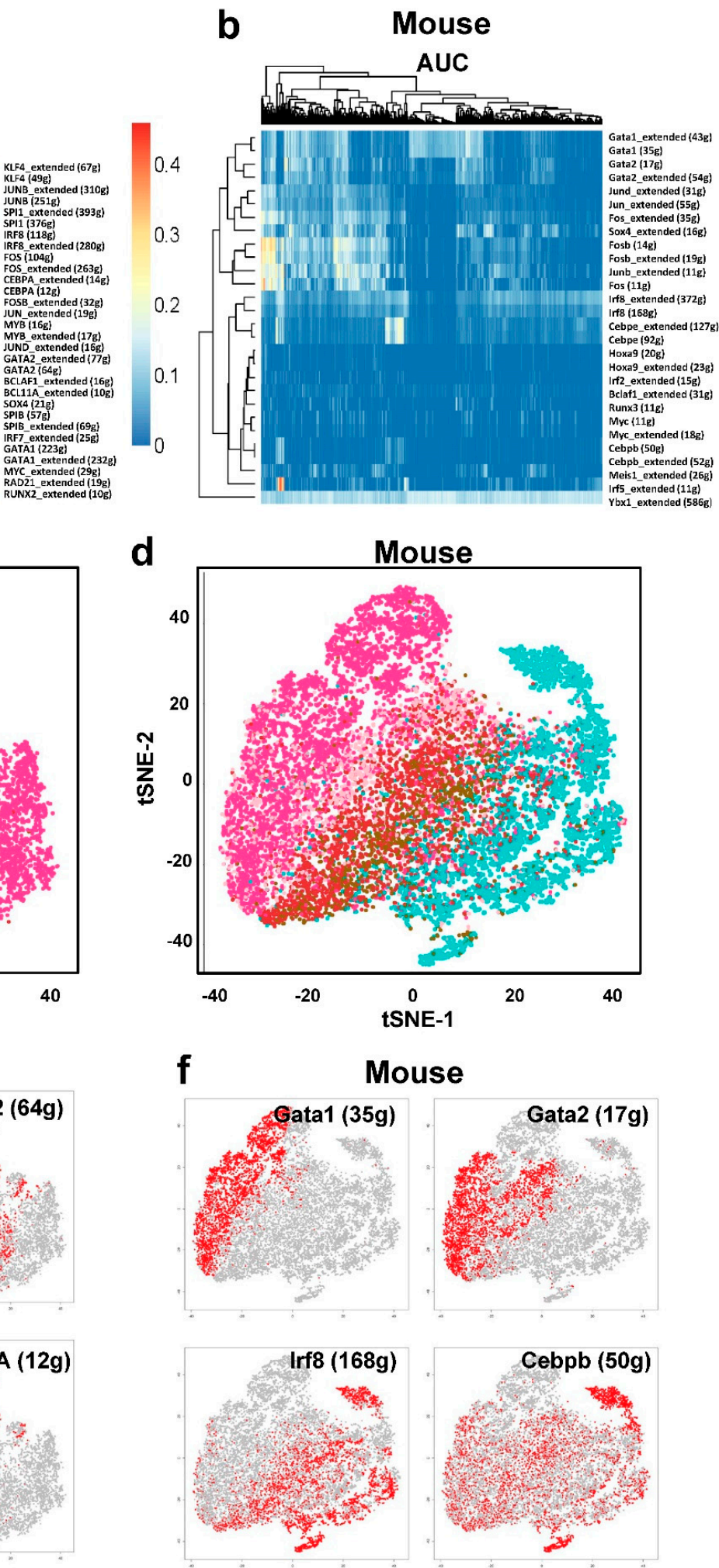

Figure 5. (a) A heatmap of the AUCell scores for the activities of regulons in each cell of human; the regulators for same cell populations were clustered together. Right are transcription factor and number of target genes. (b) A heatmap of the AUCell scores for the activities of regulons in each cell of mouse, the regulators for same cell populations were clustered together. (c) Binary regulon activity scores based tSNE plots for human (t-SNE was run with the binary regulon activity matrix as input). Each dot is a cell, which is colored by cell types. (d) Binary regulon activity scores based tSNE plots for mouse. Each dot is a cell, which is colored by cell types. (e,f) Same as (c,d), but cells are colored by AUCell scores of regulons, with red meaning high AUCell scores. 


\section{Discussion}

Our comparative transcriptomic analysis of the hematopoietic system revealed evolutionary conservation in the hematopoietic hierarchy across human and mouse. We found that HSPC compartments in the two species were composed of populations characterized by lineage-specific regulators. The lineage differentiation patterns and transcriptional profiling were well conserved between human and mouse, indicating evolutionary similarity in their hematopoietic systems. Further, we examined the TF activities that may contribute in maintaining differentiation during hematopoiesis and linked those to target genes [41]. A set of TF regulons, defined by TF-to-target correlation and TF motif analysis, was identified for different cell populations, and the regulons of human and mouse are highly conserved.

In comparing human and murine transcriptomes in hematopoiesis, one challenge is a lack of appropriate, species-matched reference gene lists, which are needed to assign cell type; the complicated hierarchical relationships between cell lineages exacerbates this problem [42]. Although characterization of the transcriptional status of individual cells enables imputation of differentiation trajectories, scRNA-seq measurements are limited by large fractions of dropouts. A new algorithm to impute dropout, and technology to increase coverage, help to improve the accuracy of trajectory inference. the integration of different types of data is a further advance. A recent study applied scRNA-seq and scATAC-seq data from bone marrow to gain deeper insight into differentiation trajectories [43]. Species conservation of a differential trajectory should be better elucidated when scRNA-seq and scATAC-seq data are available for both human and mouse.

We used regulon's activities to elucidate cellular differentiation lineages, and the result supports computational approaches to analyzing gene activation in comparing species. There is a smaller batch effect in TF regulon activation than in gene expression in single cell data, so that conclusions concerning network activity are robust and can be exploited to overcome batch or technical effects [18]; further, cell alignment is unnecessary, which is challenging due to computational complexity when integrating mega-scale single cell datasets in the future [16]. A gene regulation-based approach is a good complement to expression analysis on single cell data.

When we calculated motif enrichment and estimated regulon activities, gene regulation showed lineage conservation between human and mouse. scATAC-seq provides a more direct measurement of genome-wide activity of enhancers and promoters [44]. Chromatin accessibility is also evolutionarily conserved [45]. Thus, a joint measurement of gene expression and chromatin accessibility of the same cells in human and mouse will enable a deep comparison of the regulatory and transcriptomic landscape of hematopoiesis [46]. The current study only analyzed protein coding genes; inclusion of miRNA and lncRNA will add more layers of complexity to gene regulation to assess species conservation of haematopoiesis $[15,47,48]$.

We also downloaded the datasets from GSE81682 (mouse) and The Human Cell Atlas (human), and analyzed them with comparable computational strategies [2,49]. Similar results were obtained when we compared hematopoietic transcriptome between human and mouse. Part of our results, including the cell lineage assignment and differential trajectories, are shown in Figure S6. The cells in these two datasets follow the same differentiation trajectories as those obtained with our datasets. The results of our and third-party datasets showed that the conservation of gene regulation resulted in the similarity of gene expression in human and mouse. Transcription similarity can help in guiding the exploration of human physiological and pathological hematopoiesis with mouse models [50]. 
Supplementary Materials: The following are available online at https:/ / www.mdpi.com/article/10 .3390 / cells10050973/s1, Figure S1: tSNE plots of HSPCs from human and mouse before and after alignment, Figure S2: Typical genes and their expression in cell populations, Figure S3: Expression of characteristic genes for lineages, Figure S4: Monocle 2 identifies major branches of human and mouse hematopoiesis, Figure S5: Shared targets between regulons from human and mouse, Figure S6: tSNE plots and trajectories from human cell atlas and GSE81682, Supplementary File 1: cell type specific genes, Supplementary File 2: regulatory motifs, sequence logos and their corresponding cell types, Supplementary File 3: identified regulons by SCENIC with gmt format, Supplementary File 4: cell type specificity of regulons, Supplementary File 5: functional annotations of regulons.

Author Contributions: Conceptualization, S.G. and N.S.Y.; methodology, S.G.; analysis, S.G.; writing and editing, S.G., J.K., L.M., Z.W., X.F., S.K. and N.S.Y. All authors have read and agreed to the published version of the manuscript.

Funding: This research was funded by National Heart, Lung, and Blood Institute [Intramural Research Program].

Institutional Review Board Statement: Bone marrow samples were obtained from individuals after written informed consent in accordance with the Declaration of Helsinki, enrolled as controls under protocol NCT00001620 at the National Heart, Lung, and Blood Institute, and approved by the institutional review boards of the National Heart, Lung, and Blood Institute. All animal studies were approved by Institutional Animal Care and Use Committees at the National Heart, Lung, and Blood Institute.

Informed Consent Statement: Written informed consent was obtained from all subjects before the study.

Data Availability Statement: The datasets generated and analyzed during the current study are available in the GEO repository, with accession numbers GSE135194 and GSE142235.

Acknowledgments: We acknowledge the Biowulf PC/Linux cluster at the NIH, (http:/ /biowulf.nih. gov) used for the data analysis.

Conflicts of Interest: The authors declare no conflict of interest.

\section{References}

1. Laurenti, E.; Doulatov, S.; Zandi, S.; Plumb, I.; Chen, J.; April, C.; Fan, J.B.; Dick, J.E. The transcriptional architecture of early human hematopoiesis identifies multilevel control of lymphoid commitment. Nat. Immunol. 2013, 14, 756-763. [CrossRef] [PubMed]

2. Nestorowa, S.; Hamey, F.K.; Pijuan Sala, B.; Diamanti, E.; Shepherd, M.; Laurenti, E.; Wilson, N.K.; Kent, D.G.; Gottgens, B. A single-cell resolution map of mouse hematopoietic stem and progenitor cell differentiation. Blood 2016, 128, e20-e31. [CrossRef]

3. Zhao, X.; Gao, S.; Wu, Z.; Kajigaya, S.; Feng, X.; Liu, Q.; Townsley, D.M.; Cooper, J.; Chen, J.; Keyvanfar, K.; et al. Single-cell RNA-seq reveals a distinct transcriptome signature of aneuploid hematopoietic cells. Blood 2017, 130, 2762-2773. [CrossRef]

4. Buenrostro, J.D.; Corces, M.R.; Lareau, C.A.; Wu, B.; Schep, A.N.; Aryee, M.J.; Majeti, R.; Chang, H.Y.; Greenleaf, W.J. Integrated Single-Cell Analysis Maps the Continuous Regulatory Landscape of Human Hematopoietic Differentiation. Cell 2018, 173, 1535-1548.e16. [CrossRef]

5. Notta, F.; Doulatov, S.; Laurenti, E.; Poeppl, A.; Jurisica, I.; Dick, J.E. Isolation of single human hematopoietic stem cells capable of long-term multilineage engraftment. Science 2011, 333, 218-221. [CrossRef]

6. Notta, F.; Zandi, S.; Takayama, N.; Dobson, S.; Gan, O.I.; Wilson, G.; Kaufmann, K.B.; McLeod, J.; Laurenti, E.; Dunant, C.F.; et al. Distinct routes of lineage development reshape the human blood hierarchy across ontogeny. Science 2016, 351, aab2116. [CrossRef]

7. Baron, M.; Veres, A.; Wolock, S.L.; Faust, A.L.; Gaujoux, R.; Vetere, A.; Ryu, J.H.; Wagner, B.K.; Shen-Orr, S.S.; Klein, A.M.; et al. A Single-Cell Transcriptomic Map of the Human and Mouse Pancreas Reveals Inter- and Intra-cell Population Structure. Cell Syst. 2016, 3, 346-360.e4. [CrossRef] [PubMed]

8. Yu, Z.; Liao, J.; Chen, Y.; Zou, C.; Zhang, H.; Cheng, J.; Liu, D.; Li, T.; Zhang, Q.; Li, J.; et al. Single-Cell Transcriptomic Map of the Human and Mouse Bladders. J. Am. Soc. Nephrol. 2019, 30, 2159-2176. [CrossRef]

9. Shami, A.N.; Zheng, X.; Munyoki, S.K.; Ma, Q.; Manske, G.L.; Green, C.D.; Sukhwani, M.; Orwig, K.E.; Li, J.Z.; Hammoud, S.S. Single-Cell RNA Sequencing of Human, Macaque, and Mouse Testes Uncovers Conserved and Divergent Features of Mammalian Spermatogenesis. Dev. Cell 2020, 54, 529-547.e12. [CrossRef] [PubMed]

10. Hodge, R.D.; Bakken, T.E.; Miller, J.A.; Smith, K.A.; Barkan, E.R.; Graybuck, L.T.; Close, J.L.; Long, B.; Johansen, N.; Penn, O.; et al. Conserved cell types with divergent features in human versus mouse cortex. Nature 2019, 573, 61-68. [CrossRef]

11. Lai, S.; Huang, W.; Xu, Y.; Jiang, M.; Chen, H.; Cheng, C.; Lu, Y.; Huang, H.; Guo, G.; Han, X. Comparative transcriptomic analysis of hematopoietic system between human and mouse by Microwell-seq. Cell Discov. 2018, 4, 34. [CrossRef] 
12. Shay, T.; Jojic, V.; Zuk, O.; Rothamel, K.; Puyraimond-Zemmour, D.; Feng, T.; Wakamatsu, E.; Benoist, C.; Koller, D.; Regev, A.; et al. Conservation and divergence in the transcriptional programs of the human and mouse immune systems. Proc. Natl. Acad. Sci. USA 2013, 110, 2946-2951. [CrossRef]

13. Gao, S.; Wu, Z.; Feng, X.; Kajigaya, S.; Wang, X.; Young, N.S. Comprehensive network modeling from single cell RNA sequencing of human and mouse reveals well conserved transcription regulation of hematopoiesis. BMC Genom. 2020, 21 (Suppl. 11), 849. [CrossRef]

14. Chen, J.; Zhang, S.; Feng, X.; Wu, Z.; Dubois, W.; Thovarai, V.; Ahluwalia, S.; Gao, S.; Chen, J.; Peat, T.; et al. Conventional Co-Housing Modulates Murine Gut Microbiota and Hematopoietic Gene Expression. Int. J. Mol. Sci. 2020, 21, 6143. [CrossRef]

15. Wu, Z.; Gao, S.; Diamond, C.; Kajigaya, S.; Chen, J.; Shi, R.; Palmer, C.; Hsu, A.P.; Calvo, K.R.; Hickstein, D.D.; et al. Sequencing of RNA in single cells reveals a distinct transcriptome signature of hematopoiesis in GATA2 deficiency. Blood Adv. 2020, 4, 2656-2670. [CrossRef]

16. Butler, A.; Hoffman, P.; Smibert, P.; Papalexi, E.; Satija, R. Integrating single-cell transcriptomic data across different conditions, technologies, and species. Nat. Biotechnol. 2018, 36, 411-420. [CrossRef]

17. Guo, M.; Wang, H.; Potter, S.S.; Whitsett, J.A.; Xu, Y. SINCERA: A Pipeline for Single-Cell RNA-Seq Profiling Analysis. PLoS Comput. Biol. 2015, 11, e1004575. [CrossRef]

18. Aibar, S.; Gonzalez-Blas, C.B.; Moerman, T.; Huynh-Thu, V.A.; Imrichova, H.; Hulselmans, G.; Rambow, F.; Marine, J.C.; Geurts, P.; Aerts, J.; et al. SCENIC: Single-cell regulatory network inference and clustering. Nat. Methods 2017, 14, 1083-1086. [CrossRef]

19. Alexa, A.; Rahnenfuhrer, J.; Lengauer, T. Improved scoring of functional groups from gene expression data by decorrelating GO graph structure. Bioinformatics 2006, 22, 1600-1607. [CrossRef]

20. Shannon, P.; Markiel, A.; Ozier, O.; Baliga, N.S.; Wang, J.T.; Ramage, D.; Amin, N.; Schwikowski, B.; Ideker, T. Cytoscape: A software environment for integrated models of biomolecular interaction networks. Genome Res. 2003, 13, 2498-2504. [CrossRef]

21. Doulatov, S.; Notta, F.; Laurenti, E.; Dick, J.E. Hematopoiesis: A human perspective. Cell Stem Cell 2012, 10, 120-136. [CrossRef] [PubMed]

22. Kasraie, S.; Werfel, T. Role of macrophages in the pathogenesis of atopic dermatitis. Mediat. Inflamm. 2013, $2013,942375$. [CrossRef] [PubMed]

23. Sonnhammer, E.L.; Ostlund, G. InParanoid 8: Orthology analysis between 273 proteomes, mostly eukaryotic. Nucleic Acids Res. 2015, 43, D234-D239. [CrossRef]

24. Han, X.; Zhou, Z.; Fei, L.; Sun, H.; Wang, R.; Chen, Y.; Chen, H.; Wang, J.; Tang, H.; Ge, W.; et al. Construction of a human cell landscape at single-cell level. Nature 2020, 581, 303-309. [CrossRef] [PubMed]

25. Kiselev, V.Y.; Yiu, A.; Hemberg, M. scmap: Projection of single-cell RNA-seq data across data sets. Nat. Methods 2018, 15, 359-362. [CrossRef]

26. Saelens, W.; Cannoodt, R.; Todorov, H.; Saeys, Y. A comparison of single-cell trajectory inference methods. Nat. Biotechnol. 2019, 37, 547-554. [CrossRef]

27. Qiu, X.; Hill, A.; Packer, J.; Lin, D.; Ma, Y.A.; Trapnell, C. Single-cell mRNA quantification and differential analysis with Census. Nat. Methods 2017, 14, 309-315. [CrossRef]

28. Suzuki, M.; Kobayashi-Osaki, M.; Tsutsumi, S.; Pan, X.; Ohmori, S.; Takai, J.; Moriguchi, T.; Ohneda, O.; Ohneda, K.; Shimizu, R.; et al. GATA factor switching from GATA2 to GATA1 contributes to erythroid differentiation. Genes Cells 2013, 18, 921-933. [CrossRef]

29. Qiu, X.; Mao, Q.; Tang, Y.; Wang, L.; Chawla, R.; Pliner, H.A.; Trapnell, C. Reversed graph embedding resolves complex single-cell trajectories. Nat. Methods 2017, 14, 979-982. [CrossRef] [PubMed]

30. Setty, M.; Kiseliovas, V.; Levine, J.; Gayoso, A.; Mazutis, L.; Pe'er, D. Characterization of cell fate probabilities in single-cell data with Palantir. Nat. Biotechnol. 2019, 37, 451-460. [CrossRef]

31. Hoppe, P.S.; Schwarzfischer, M.; Loeffler, D.; Kokkaliaris, K.D.; Hilsenbeck, O.; Moritz, N.; Endele, M.; Filipczyk, A.; Gambardella, A.; Ahmed, N.; et al. Early myeloid lineage choice is not initiated by random PU.1 to GATA1 protein ratios. Nature 2016, 535, 299-302. [CrossRef] [PubMed]

32. Ferreira, R.; Ohneda, K.; Yamamoto, M.; Philipsen, S. GATA1 function, a paradigm for transcription factors in hematopoiesis. Mol. Cell. Biol. 2005, 25, 1215-1227. [CrossRef] [PubMed]

33. Unnisa, Z.; Clark, J.P.; Roychoudhury, J.; Thomas, E.; Tessarollo, L.; Copeland, N.G.; Jenkins, N.A.; Grimes, H.L.; Kumar, A.R. Meis1 preserves hematopoietic stem cells in mice by limiting oxidative stress. Blood 2012, 120, 4973-4981. [CrossRef]

34. Zargari, S.; Negahban Khameneh, S.; Rad, A.; Forghanifard, M.M. MEIS1 promotes expression of stem cell markers in esophageal squamous cell carcinoma. BMC Cancer 2020, 20, 789. [CrossRef]

35. Papoudou-Bai, A.; Hatzimichael, E.; Barbouti, A.; Kanavaros, P. Expression patterns of the activator protein-1 (AP-1) family members in lymphoid neoplasms. Clin. Exp. Med. 2017, 17, 291-304. [CrossRef]

36. Fan, F.; Bashari, M.H.; Morelli, E.; Tonon, G.; Malvestiti, S.; Vallet, S.; Jarahian, M.; Seckinger, A.; Hose, D.; Bakiri, L.; et al. The AP-1 transcription factor JunB is essential for multiple myeloma cell proliferation and drug resistance in the bone marrow microenvironment. Leukemia 2017, 31, 1570-1581. [CrossRef]

37. Sandler, V.M.; Lis, R.; Liu, Y.; Kedem, A.; James, D.; Elemento, O.; Butler, J.M.; Scandura, J.M.; Rafii, S. Reprogramming human endothelial cells to haematopoietic cells requires vascular induction. Nature 2014, 511, 312-318. [CrossRef] 
38. Guo, H.; Barberi, T.; Suresh, R.; Friedman, A.D. Progression from the Common Lymphoid Progenitor to B/Myeloid PreproB and ProB Precursors during B Lymphopoiesis Requires C/EBPalpha. J. Immunol. 2018, 201, 1692-1704. [CrossRef]

39. Bhullar, J.; Sollars, V.E. YBX1 expression and function in early hematopoiesis and leukemic cells. Immunogenetics 2011, 63, 337-350. [CrossRef]

40. Yokoyama, H.; Harigae, H.; Takahashi, S.; Furuyama, K.; Kaku, M.; Yamamoto, M.; Sasaki, T. Regulation of YB-1 gene expression by GATA transcription factors. Biochem. Biophys. Res. Commun. 2003, 303, 140-145. [CrossRef]

41. Hao, S.; Yan, K.K.; Ding, L.; Qian, C.; Chi, H.; Yu, J. Network Approaches for Dissecting the Immune System. Iscience 2020, $23,101354$.

42. Lahnemann, D.; Koster, J.; Szczurek, E.; McCarthy, D.J.; Hicks, S.C.; Robinson, M.D.; Vallejos, C.A.; Campbell, K.R.; Beerenwinkel, N.; Mahfouz, A.; et al. Eleven grand challenges in single-cell data science. Genome Biol. 2020, 21, 31. [CrossRef]

43. Ranzoni, A.M.; Tangherloni, A.; Berest, I.; Riva, S.G.; Myers, B.; Strzelecka, P.M.; Xu, J.; Panada, E.; Mohorianu, I.; Zaugg, J.B.; et al. Integrative Single-Cell RNA-Seq and ATAC-Seq Analysis of Human Developmental Hematopoiesis. Cell Stem Cell 2021, 28, 472-487.e7. [CrossRef]

44. Bravo Gonzalez-Blas, C.; Minnoye, L.; Papasokrati, D.; Aibar, S.; Hulselmans, G.; Christiaens, V.; Davie, K.; Wouters, J.; Aerts, S. cisTopic: Cis-regulatory topic modeling on single-cell ATAC-seq data. Nat. Methods 2019, 16, 397-400. [CrossRef] [PubMed]

45. Minnoye, L.; Taskiran, I.I.; Mauduit, D.; Fazio, M.; Van Aerschot, L.; Hulselmans, G.; Christiaens, V.; Makhzami, S.; Seltenhammer, M.; Karras, P.; et al. Cross-species analysis of enhancer logic using deep learning. Genome Res. 2020, 30, 1815-1834. [CrossRef]

46. Cao, J.; Cusanovich, D.A.; Ramani, V.; Aghamirzaie, D.; Pliner, H.A.; Hill, A.J.; Daza, R.M.; McFaline-Figueroa, J.L.; Packer, J.S.; Christiansen, L.; et al. Joint profiling of chromatin accessibility and gene expression in thousands of single cells. Science 2018, 361, 1380-1385. [CrossRef]

47. Yang, Q.; Wan, Q.; Zhang, L.; Li, Y.; Zhang, P.; Li, D.; Feng, C.; Yi, F.; Zhang, L.; Ding, X.; et al. Analysis of LncRNA expression in cell differentiation. RNA Biol. 2018, 15, 413-422. [CrossRef]

48. Shim, J.; Nam, J.W. The expression and functional roles of microRNAs in stem cell differentiation. BMB Rep. 2016, 49, 3-10. [CrossRef]

49. Rozenblatt-Rosen, O.; Stubbington, M.J.T.; Regev, A.; Teichmann, S.A. The Human Cell Atlas: From vision to reality. Nature 2017, 550, 451-453. [CrossRef]

50. Saito, Y.; Shultz, L.D.; Ishikawa, F. Understanding Normal and Malignant Human Hematopoiesis Using Next-Generation Humanized Mice. Trends Immunol. 2020, 41, 706-720. [CrossRef] 